\title{
Neuronale Kontrolle bei chronisch entzündlichen und obstruktiven Lungenerkrankungen wie Asthma bronchiale und COPD
}

\section{Innervation of the Airways in Asthma Bronchiale and Chronic Obstructive Pulmonary Disease (COPD)}

Autoren

Institute
Q. T. Dinh ${ }^{1,2}$, H. Suhling ${ }^{1}$, A. Fischer ${ }^{3}$, A. Braun ${ }^{4}$, T. Welte ${ }^{1}$

Die Institutsangaben sind am Ende des Beitrags gelistet. eingereicht 27.10.2010 akzeptiert nach Revision 8. 12.2010

\section{Bibliografie}

Dol http://dx.doi.org/ 10.1055/s-0030-1256123

Online-Publikation: 26.1.2011

Pneumologie 2011; 65:

283-292 @ Georg Thieme

Verlag KG Stuttgart · New York ISSN 0934-8387

\section{Korrespondenzadresse} PD Dr. med. Quoc Thai Dinh Klinik für Pneumologie Leiter der Arbeitsgruppe: Experimentelle Pneumologie und Allergologie Medizinische Hochschule Hannover (MHH) Carl-Neuberg-Str. 1 30625 Hannover Dinh.Quoc@mh-hannover.de

\section{Zusammenfassung \\ $\nabla$}

Chronisch entzündliche Atemwegserkrankungen wie Asthma bronchiale und die chronisch obstruktive Lungenerkrankung (COPD) können nach den gegenwärtigen Forschungsergebnissen weder als eine rein immunologische noch als eine ausschließlich neuronale Erkrankung angesehen werden $[1,2]$. Die entzündlichen Veränderungen werden von einer Vielzahl an immunologischen und neuronalen Mediatoren hervorgerufen und beeinflusst. Im Bereich der Pathophysiologie und Pathobiochemie des Asthma bronchiale sowie der COPD sind bereits über fünfzig Mediatoren mit verschiedenen Effekten verschiedenste pulmonale Funktionen beschrieben worden. Die Mediatoren werden dabei von Entzündungszellen, wie Mastzellen, Eosinophilen, Basophilen, Neutrophilen oder T-Lymphozyten, gebildet. Weiterhin gehören auch andere Zellen, wie Epithelzellen, Endothelzellen, Myozyten oder Atemwegsneurone, zu den Mediator-bildenden und freisetzenden Zellen. Neben den klassischen Mediatoren Noradrenalin in postganglionären sympathischen Nervenfasern und Acetylcholin in parasympathischen Nervenfasern existiert eine Reihe von Neuropeptiden, die ausgeprägte pharmakologische Effekte auf den Muskeltonus der Blutgefäße und der Bronchien, die Drüsenfunktion und auf Entzündungs- und Immunzellen hat. Diese Neuropeptide gehören zu keinem morphologisch eingrenzbaren System. Diese Neuropeptide werden unter dem Begriff des nicht-adrenergen nicht-cholinergen (NANC)-Systems zusammengefasst. Die Rolle des Nervensystems in Bezug auf die asthmatische oder chronisch obstruktive Erkrankung wurde bisher sehr unterschiedlich gewichtet und bewertet. Sehr früh begann man sich für das Nervensystem der menschlichen Lunge $\mathrm{zu}$ interessieren und es anatomisch detailliert zu beschreiben [3-5], da ein Zusammenhang zwischen dem Nervensystem und der

\section{Abstract \\ $\nabla$}

Airway nerves have the capacity to control airway functions via neuronal reflexes and through neuromediators and neuropeptides. Neuronal mechanisms are known to play a key role in the initiation and modulation of airway hyperresponsiveness and inflammation. Therefore, the nerve fibres may contribute to airway narrowing in asthma and COPD. In addition to the traditional transmitters such as norepinephrine in postganglionic sympathetic nerve fibres and acetylcholine in parasympathetic nerve fibres, a large number of neuropeptides have been identified to have different pharmacological effects on the muscle tone of the vessels and bronchi, mucus secretion and immune cells. Meanwhile, a broad range of stimuli including capsaicin, bradykinin, hyperosmolar saline, tobacco smoke, allergens, ozone, inflammatory mediators and even cold, dry air have been shown to activate sensory nerve fibres to release neuropeptides such as the tachykinins substance $\mathrm{P}$ (SP) and neurokinin $\mathrm{A}$ (NKA) to mediate neurogenic inflammation. Different aspects of the neurogenic inflammation have been well studied in animal models of chronic airway inflammation and anticholinergic agents such as ipratropium bromide (Atrovent ${ }^{\circledR}$ ) and tiotropium bromide (Spiriva ${ }^{\circledR}$ ) have been proved to be important when used as bronchodilators for the treatment of obstructive airway diseases such as COPD. However, little is known about the role of neurogenic airway inflammation in human diseases. In this review, we address the current knowledge of the airway sensory nerves in human asthma and COPD. 
Pathophysiologie des Asthma bronchiale und der COPD vermutet wurde. Seitdem wurden unterschiedliche Aspekte des Nervensystems untersucht. Etablierte Pharmakotherapiekonzepte mit Anticholinergika, wie Ipratropiumbromid (Atrovent ${ }^{\circledR}$ ) oder Tiotropiumbromid (Spiriva ${ }^{\circledR}$ ), bestehen darin, durch einen kompetitiven Antagonismus die Effekte des natürlichen Überträgerstoffes an den cholinergen Neuronen zu hemmen und damit die Erschlaffung der glatten Muskulatur der Bronchien zu erreichen. Die effektivsten Bronchodilatatoren sind die 32 -Sympathomimetika (Sabutamol), welche effektiv die Freisetzung von Acetylcholin aus cholinergen Neuronen hemmen und gleichzeitig die $\beta 2-$ Rezeptoren an den motorischen Endplatten der Muskelzellen stimulieren. Dieser Wirkungsmechanismus führt zu einer Bronchodilatation durch Relaxation der glatten Muskulatur. Über die weitere Rolle des Nervensystems bei den chronisch obstruktiven Lungenerkrankungen des Menschen ist aber bisher nur wenig bekannt. Wegen der Komplexität der neuroimmunologischen Interaktion beim Asthma bronchiale und der COPD muss in $\mathrm{Zu}-$ kunft weitere Forschung zum Verständnis der Rolle der Atemwegsinnervation und deren Aktivierung und Interaktion mit Entzündungszellen unternommen werden. Dieser Artikel gibt eine Übersicht über die bisherigen Erkenntnisse der neuronalen Kontrolle der beiden großen obstruktiven Atemwegserkrankungen.

\begin{tabular}{|c|c|}
\hline \multicolumn{2}{|c|}{$\begin{array}{l}\text { Abkürzungsverzeichnis } \\
\nabla\end{array}$} \\
\hline $\mathrm{ACh}$ & Acetylcholin \\
\hline AChE & ACh-Abbauenzym Acetylcholinesterase \\
\hline CGRP & Calcitonin Gene-verwandtes Peptid \\
\hline ChAT & Cholin Acetyltransferase \\
\hline COPD & chronisch obstruktive Lungenerkrankung \\
\hline DC & Dendritische Zelle \\
\hline eNANC & $\begin{array}{l}\text { exzitatorisches nicht-adrenerges- } \\
\text { nicht-cholinerges System }\end{array}$ \\
\hline iNANC & $\begin{array}{l}\text { inhibitorisches nicht-adrenerges } \\
\text { nicht-cholinerges System }\end{array}$ \\
\hline NANC & nicht-adrenerges-nicht-cholinerges System \\
\hline NA & Noradrenalin \\
\hline NKA & Neurokinin A \\
\hline NKB & Neurokinin B \\
\hline NK-1-Rezeptor & Neurokinin-1-Rezeptor \\
\hline NK-2-Rezeptor & Neurokinin-2-Rezeptor \\
\hline NK-3-Rezeptor & Neurokinin-3-Rezeptor \\
\hline NPY & Neuropeptid Tyrosin \\
\hline NO & Stickstoffmonoxid \\
\hline NOS & NO-Synthase \\
\hline PGP 9.5 & Proteingenproduct 9.5 \\
\hline PPT-A-Gen & Präprotachykinin-A-Gen \\
\hline PPT-B-Gen & Präprotachykinin-B-Gen \\
\hline \multicolumn{2}{|c|}{ p75 Neurotrophin } \\
\hline Rezeptor & Neurotrophin Rezeptor p75 \\
\hline RAR & schnell adaptierende Dehnungsrezeptoren \\
\hline SCG & Superior cervical ganglion \\
\hline SP & Substanz P \\
\hline $\mathrm{TH}$ & Tyrosin Hydroxylase \\
\hline TRPV1 & Transient Rezeptor Potential Vanilloid 1 \\
\hline trkA & Tyrosin Kinase Rezeptor trkA \\
\hline $\operatorname{trkB}$ & Tyrosin Kinase Rezeptor trkB \\
\hline VIP & Vasoactives Intestinales Polypeptid \\
\hline VR1 & Vanilloid Rezeptor 1 \\
\hline
\end{tabular}

\section{Die Innervation der Atemwege und der Lungen} $\nabla$

\section{Sympathische Innervation}

Das Zentrum der sympathischen Innervation der unteren Atemwege und der Lunge liegt im Nucleus intermediolaterales und im Nucleus intercalatus des thorakalen Rückenmarks. Axone der sympathischen präganglionären Neurone verlassen gemeinsam mit allen motorischen Fasern über die Vorderwurzel das Rückenmark und ziehen zu den prä- und paravertebralen Grenzstrangganglien, hauptsächlich zu dem Ganglion cervicale superius und medius sowie zu dem Ganglion stellatum. Von hier ziehen postganglionäre sympathische Axone über den R. pulmonales zur Lunge. Alle die Lunge versorgenden Nerven treten am Hilus in die Lunge ein und folgen den Bronchien und Gefäßen [6].

Sympathische Nervenfasern konnten um die tracheobronchialen und pulmonalen Gefäße und die exokrinen Drüsen der unteren Atemwege bei verschiedenen Spezies wie Meerschweinchen, Ratte, Maus, Affe und Mensch nachgewiesen werden [7]. Dabei ist die sympathische Innervation der glatten Atemwegsmuskulatur bei den Spezies unterschiedlich ausgeprägt. Bei Meerschweinchen wurde eine dichte Innervation an sympathischen Nerven gefunden, während bei Affen nur eine spärliche Versorgung der glatten Atemwegsmuskulatur mit sympathischen Nervenfasern beobachtet wurde [8]. Im Gegensatz zu seinem Gegenspieler, dem Parasympathikus, hat der Sympathikus nach heutigem Wissensstand eine untergeordnete Bedeutung. Nur tracheobronchiale Blutgefäße werden direkt sympathisch innerviert, die glatte Atemwegsmuskulatur weist allerdings auch $\beta$-Rezeptoren auf [9]. Da die glatte Atemwegsmuskulatur eine funktionelle Einheit bildet, sind wahrscheinlich nur einzelne Kontakte zwischen glatten Muskelzellen mit sympathischen Nervenfasern erforderlich, um eine Modulation der bronchialen Atemwegsmuskulatur auszulösen. Es kann keine funktionelle Relevanz der spärlichen sympathischen Innervation der Atemwege des Menschen angenommen werden [10]. Das Verteilungmuster der $\beta$-Rezeptoren in den Atemwegen ist ebenfalls wichtig im Hinblick auf die Pharmakotherapie mit $\beta 2$-Mimetika als wichtigste Bronchodilatatoren bei der Behandlung des Asthma bronchiale. Die Stimulation von sympathischen Nervenfasern führt über die Aktivierung von $\beta$-Rezeptoren zu einer Erweiterung der Bronchien [11]. Es wurde z.B. in Patienten nach Unterbrechung des Sympathikus (thorakale Sympathektomie) eine gesteigerte bronchiale Hyperreagibilität gemessen [12]. In Studien zur Untersuchung der Verteilung und Dichte der $\beta$-Rezeptoren konnte eine hohe Dichte beim Menschen im Lungengewebe nachgewiesen werden. Auch andere Spezies wiesen diesen Befund auf. Die Dichte der $\beta$-Rezeptoren nimmt von der Trachea bis in die terminalen Bronchiolen $\mathrm{zu}$, während nur vereinzelte $\alpha$-Rezeptoren in den Atemwegen lokalisiert wurden [13].

Sympathische Nervenfasern enthalten neben dem klassischen Transmitter Noradrenalin (NA) weitere Neuropeptide wie Neuropeptid Tyrosin (NPY), Stickstoffmonoxid (NO) und Vasoaktives Intestinales Polypeptid (VIP) als postganglionäre Mediatoren. NPY wurde zusammen mit NA in sympathischen Nervenfasern [5] um Arterien, Arteriolen und in der glatten Bronchialmuskulatur der unteren Atemwege nachgewiesen $[14,15]$. NPY kommt teilweise gemeinsam mit NA oder mit VIP in sympathischen Atemwegsneuronen vor [16]. Bekannt ist auch, dass zahlreiche sympathische Atemwegsneurone NO produzieren [17]. Aufgrund der zahlreichen Neuromediatoren, die von sympathischen Nervenfasern synthetisiert und freigesetzt werden, wird eine wichti- 
ge Rolle der sympathischen Atemwegsinnervation sowohl unter normalen als auch unter pathologischen Bedingungen vermutet.

\section{Parasympathische Innervation}

Die präganglionären parasympathischen Neurone liegen im Nucleus dorsalis nervi vagi und im Nucleus ambiguus des Hirnstamms. Die Axone dieser Neurone ziehen zum Teil mit dem N. laryngeus recurrens $[18,19]$ zu den kleinen intramuralen Ganglien der Trachea. Ein anderer Teil erreicht die postganglionären Neurone über die vagalen Rami bronchiales [20,21]. Kurze Axone der postganglionären Neurone versorgen die glatte Muskulatur der Trachea, der Bronchien, der Bronchioli, daneben die Drüsen und die sekretorischen Zellen des Epithels sowie die großen Pulmonalgefäße der Atemwege [22]. Sympathische, parasympathische und sensible Nervenfasern vereinigen sich zu einem Nervengeflecht um die Atemwege, das an der Hinterwand der Trachea liegt und am Lungenhilus in die Lunge eintritt [23].

Klassischer Mediator der prä- und postganglionären parasympathischen Neurone ist Acetylcholin (ACh). Bei Untersuchungen mit verschiedenen Säugetierspezies konnte durch Nachweis des AChAbbauenzyms Acetylcholinesterase (AChE), dieses als Mediator in postganglionären parasympathischen Neuronen, die mit ihren Nervenendigungen die glatte Atemwegsmuskulatur und die exokrinen Drüsen innervieren, nachgewiesen werden [7]. Mit immunhistochemischen Methoden zur Lokalisation des ACh-synthetisierenden Enzyms, Cholin Acetyltransferase (ChAT) konnte gezeigt werden, dass Blutgefäße und die lamina propria der Schleimhäute der Lunge weitgehend frei sind von parasympathischen Fasern. Beim Meerschweinchen ergibt sich ein anderer Befund mit einer parasympathischen Innveration selbst in diesen Bereichen [22,24].

Neben ACh ließen sich in den intrinsischen parasympathischen Neuronen der Atemwege beim Menschen weitere Mediatoren wie VIP und NO nachweisen, sie haben unterschiedliche und teilweise entgegengesetzte pharmakologische Wirkungen [25]. Sie kommen mit ChAT teilweise kolokalisiert vor [26]. Bei anderen Spezies als dem Menschen konnten noch weitere Mediatoren wie Galanin [27], Substanz P (SP) [28, 29], Calcitonin Gene-Related Peptide [30] und Opioide [31] in Kolokalisation nachgewiesen werden. Eine Aktivierung der parasympathischen Atemwegsinnervation führt zur Bronchokonstriktion und Schleimsekretion. Dabei spielen C-Fasern des N. vagus als predominante Effektorneurone der Bronchokonstriktion eine entscheidende Rolle. Barnes et al. vertreten die Meinung, dass in der Exazerbation des Asthmas bronchiale dieser neuronale Mechanismus vermehrt aktiviert wird [32]. Unterstützt wird diese These von experimentellen Erkenntnissen, dass eine bilaterale Vagatomie in einem Asthmatiermodell die Bronchokonstriktion vermindern kann [33]. Sowohl bei Asthma wie auch bei der COPD kommt es zu einer durch Stress ausgelösten Aktivierung der vagalen C-Fasern via TRPV1-Rezeptoren durch endogene reaktive Mediatoren [34].

\section{Sensible Innervation}

Die sensible Innervation der unteren Atemwege und der Lunge verläuft im N. vagus. Sie erhält nicht nur Informationen aus Erregungen von Berührungs- und Dehnungsrezeptoren an der Trachea, den Bronchien, den Bronchiolen, sondern auch denen unter der Pleura. Nach ihren elektrophysiologischen Eigenschaften lassen sich die sensiblen Nervenfasern in drei Klassen einteilen: langsam adaptierende Dehnungsrezeptoren, schnell adaptierende Dehnungsrezeptoren und C-Faser-Rezeptoren. C-Fasern unterscheiden sich nicht nur elektrophysiologisch, sondern auch neu- roanatomisch von den Fasern der Dehnungsrezeptoren [31]. Die Perikaryen der Dehnungsrezeptoren des Meerschweinchens befinden sich im Ganglion nodosum, die der C-Fasern im Ganglion jugulare. Im Gegensatz zu den Dehnungsrezeptoren weisen C-Fasern aufgrund ihrer nicht myelinisierten Axone langsame Leitungsgeschwindigkeiten auf $(<1 \mathrm{~m} / \mathrm{s})$ [30]. Funktionell verhält sich die untere sensible Atemwegsinnervation wie die der oberen Atemwege und kann auf Reize reagieren. Stimuli wie Capsaicin, Bradykinin, hyperosmotische Salzlösung, Zigarettenrauch, Allergene, Ozon, proinflammatorische Mediatoren und kalte trockene Luft sind in der Lage, die sensible Innervation der unteren Atemwege zu aktivieren. Es kommt dann zu einer Depolarisation der sensiblen Faser. Besonderes Augenmerk richtet sich auf die C-Fasern, sie dienen nicht nur der Reizleitung der Nozizeptoren, die durch mechanische, chemische und thermische Reize aktiviert werden, sondern können auch unmittelbar nach Stimulation proinflammatorische Neuropeptide wie die Tachykinine und Calcitonin Gene-verwandtes Peptide (CGRP) freisetzen. Diese proinflammatorischen Neuropeptide bewirken in der Umgebung eine Entzündung. Es ist schon ein unterschwelliger Reiz (keine Depolarisation) ausreichend, um eine Freisetzung von Neuropeptiden zu bewirken.

Die Synthese der Neuropeptide findet im Zellkörper der sensiblen Neurone statt und über einen antidromen Transport gelangen sie durch den Dendrit in die Nervenendigungen in der Lunge. Hier werden sie nach Stimulation aus synaptischen Vesikeln [32] freigesetzt. Zur Familie der Tachykinine zählen die Neuropeptide Substance P (SP) und Neurokinin A (NKA), die eine proinflammatorische Wirkung in den Atemwegen besitzen. Ein wichtiges in der Atemwegsinnervation vorkommendes und strukturell aber mit den Tachykininen nicht verwandtes Neuropeptid ist CGRP. CGRP kommt gehäuft mit Tachykininen in sensiblen Neuronen der Atemwege vor und unterstützt die Vasodilatation in den Gefäßen der unteren Atemwege [5,36] ( $\bullet$ Abb. 1 u. 2).

Die Aktivität der sensiblen Neurone wird durch inhibitorische Rezeptoren auf der Oberfläche beeinflusst, dazu gehören Canabinoid- (CB2), Histamin- (H3), Dopamin- (D2), Adrenalin-(alpha 2) oder Opioidrezeptoren (OP3). Im Gegensatz dazu zählen TRPV1, H1- und Bradykinin B2-Rezeptoren zu den exzitatorischen Rezeptoren [11].

\section{Mediatoren und Rezeptoren in den Atemwegen \\ $\nabla$}

\section{Noradrenalin}

Klassischer Neurotransmitter der postganglionären sympathischen Nervenfasern in den unteren Atemwegen der Säuger einschließlich des Menschen ist Noradrenalin (NA). Das Ausgangssubstrat der Biosynthese von Noradrenalin und Adrenalin ist Tyrosin, das durch das Schrittmacherenzym Tyrosin-Hydroxylase (TH) in Dihydroxyphenylalanin (Dopa) und weiter in Dopamin umgewandelt wird. Durch Hydroxylierung des $\beta$-Kohlenstoffes der Seitenkette des Dopamin mit Hilfe des Enzyms Dopamin- $\beta$ Hydroxylase entsteht Noradrenalin. In einem weiteren biochemischen Reaktionsschritt entsteht durch die Methylierung des Noradrenalins Adrenalin. Diese Katecholamine werden in den Zellen des Nebennierenmarks und des sympathischen Nervensystems in Sekretgranula gespeichert und unmittelbar nach Stimulation freigesetzt.

Immunhistochemische Färbungen konnten die Expression von TH in Nervenfasern zeigen, die die Gefäße und die glatte Muskulatur der Trachea und der Bronchien der unteren Atemwege 

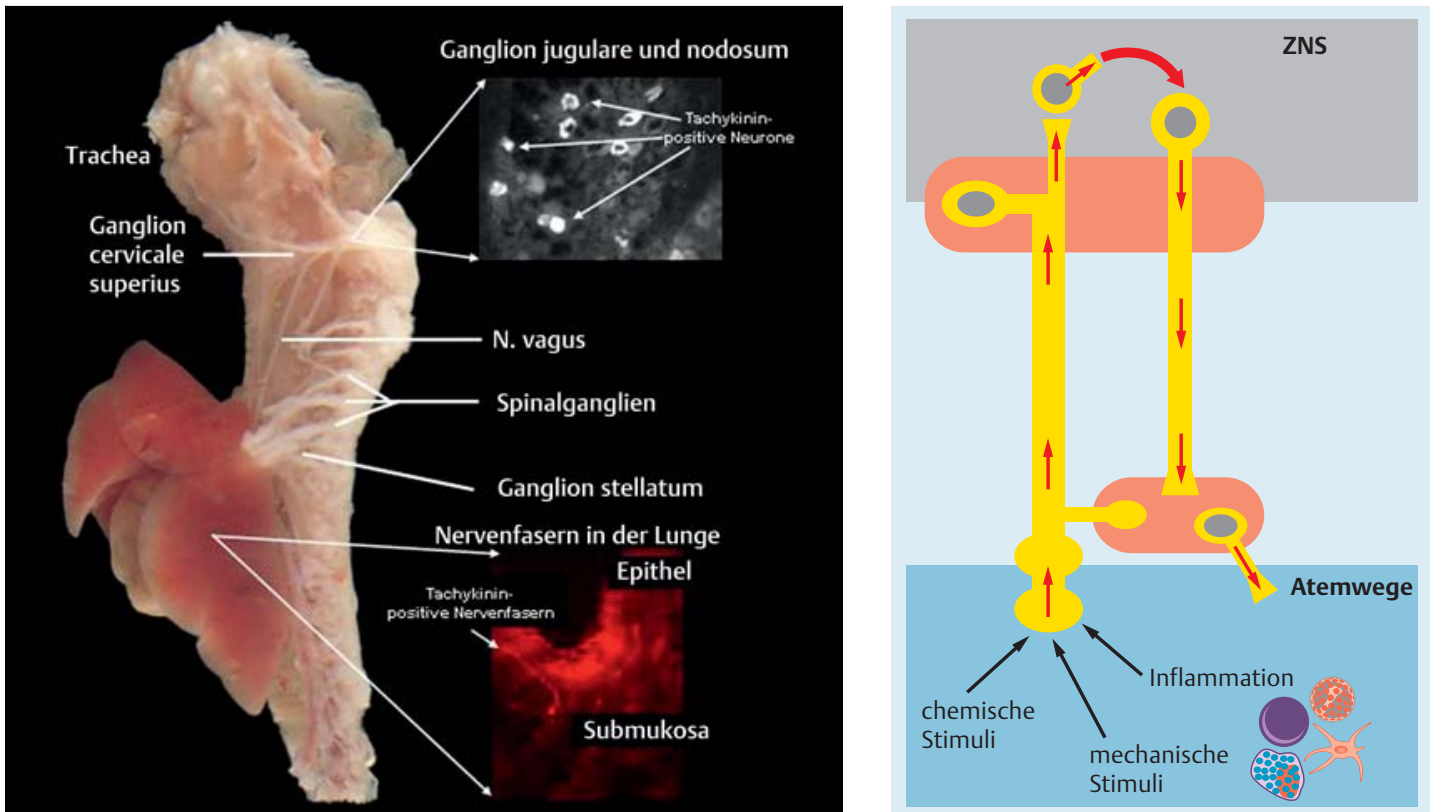

Abb. 2 Bei den sensiblen Neuronen handelt sich hier um pseudounipolare Neurone, deren Axone mit dem N. vagus verlaufen. Aktivierung sensibler Neurone durch chemische und mechanische Irritanten oder Entzündungen kann zur Biosynthese und Freisetzung von proinflammatorischen Neuropeptiden wie Tachykinine (SP/NKA) und CGRP in den Atemwegen führen.

Abb. 1 Die Neurone der sensiblen Atemwegsinnervation liegen in den vagalen sensiblen Ganglien jugulare und nodosum. Eine zusätzliche sensible Versorgung der Atemwege stammt aus den thorakalen Spinalganglien. Die Axone der spinalen sensiblen Neurone verlaufen zusammen mit den sympathischen Axonen, durchqueren die sympathischen Grenzstrangganglien und ziehen über die Hinterwurzel im Hinterhorn des Rückenmarks [95].

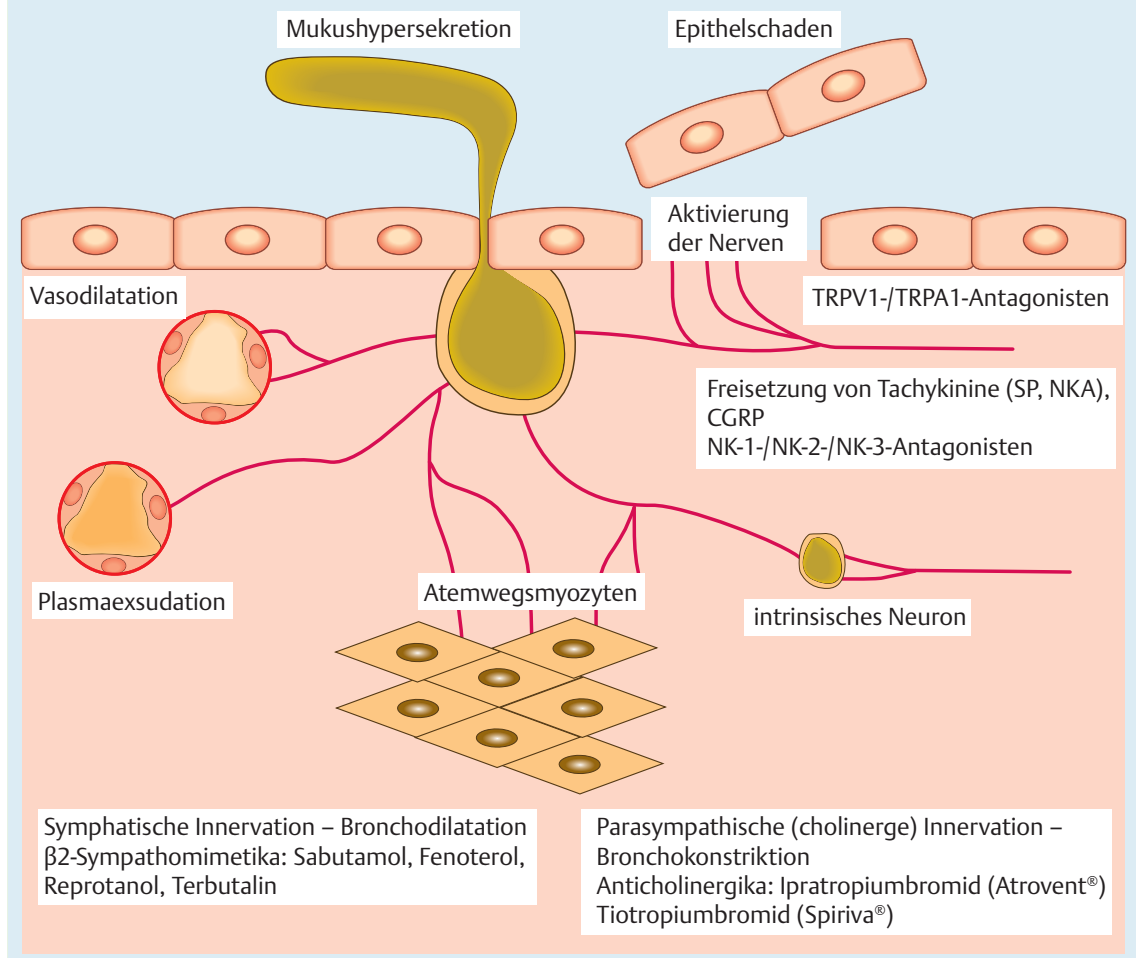

Abb. 3 Modulation der sympathischen und parasympathischen Innervation bei chronisch obstruktiven Lungenerkrankungen Asthma bronchiale und COPD.

Aktivierung der sensiblen Innervation führt zu einer Freisetzung von Neuropetiden wie Tachykinine und CGRP, die Plasmaexsudation, Vasodilatation und verstärkte Mukusekretion verursachen. Eine Reihe von $\beta 2$-Sympathomimetika, wie die Rezeptorenagonisten Sabutamol, Fenoterol, Reprotanol, Terbutalin, gehören zu den effektivsten Bronchodilatatoren. Anticholinergika, wie Ipratropiumbromid (Atrovent ${ }^{\circledR}$ ) oder Tiotropiumbromid (Spiriva ${ }^{\circledR}$ ), antagonisieren die muskurinischen ACh-Rezeptoren M3-Rezeptoren und führen zur Erschlaffung der glatten Muskulatur der Bronchien. Die Effektivität der Tachykinin-Antagonisten bei Menschen ist noch unklar. TRPV1-/TRPA1-Antagonisten können neue Therapieansätze für Asthma bronchiale und COPD eröffnen.

innervieren. Viele TH-positive Nervenfasern besitzen NPY. Mittels Tracingstudien konnte die Herkunft dieser TH-+/NPY-+-Nervenfasern nachgewiesen werden. Sie stammen hauptsächlich aus den Neuronen des sympathischen Ganglion stellatum und des Ganglion cervicale superius [5,37]. Es wurden bisher aber auch TH-positive Neurone in Vagalganglien der Ratte mit Projektion zum Ösophagus und Magen gefunden [38].
NA vermittelt vorwiegend über $\beta 2$-Rezeptoren durch eine Aktivierung der Adenylatzyklase eine Bronchodilatation, eine Sekretionssteigerung sowie eine Steigerung der Zilien-Schlagfrequenz beim Menschen [39]. 


\section{Acetylcholin}

Acetylcholin, das aus den parasympathischen Nervenendigungen freigesetzt wird, kann sich an muskarinischen ACh-Rezeptoren mit unterschiedlicher Funktion binden. Bisher sind 5 muskarinische ACh-Rezeptoren Untertypen (M1-, M2-, M3-, M4-, M5-Rezeptor) bekannt. M1-Rezeptoren sind am parasympathischen Ganglion lokalisiert. Aktivierung der M1-Rezeptoren kann die Erregungsleitung des Nervus vagus und cholinerge Reflexe steigern. M2-Rezeptoren befinden sich direkt an der motorischen Endplatte und sind sogenannte Autorezeptoren, die die Ausschüttung des Überträgerstoffes Acteylcholin im Feedback-Mechanismus regeln [40-42]. Anticholinergika, wie Ipratropiumbromid $\left(\right.$ Atrovent $^{\circledR}$ ) oder Tiotropiumbromid $\left(\right.$ Spiriva $\left.^{\circledR}\right)$, wirken durch einen kompetitiven Antagonismus des Acetylcholins an den M3-Rezeptoren der glatten Bronchialmuskulatur und wirken in den Luftwegen bronchospasmolytisch. Acetylcholin wirkt nicht nur bronchokonstriktorisch, sondern kann bei einer Exazerbation bei Patienten mit Asthma oder COPD auch zu vermehrter Schleimproduktion mit fatalen Folgen führen [43]. Seit kurzem ist auch bekannt, dass Acetylcholin nicht nur von Neuronen synthetisiert, sondern auch von anderen Zellen wie Epithel- und Immunzellen exprimiert wird [44].

\section{Mediatoren des (nicht-adrenergen nicht-cholinergen) NANC-Sytems}

Neben den bereits erwähnten Mediatoren existiert eine Reihe von Neuropeptiden, die zu keinem morphologisch eingrenzbaren Nervensystem gehören und unter dem Begriff des nichtadrenergen nicht-cholinergen (NANC)-Systems zusammengefasst werden [45].

Aufgrund physiologischer und pharmakologischer Erkenntnisse können die NANC-Mediatoren in zwei funktionelle Gruppen eingeordnet werden [46]. Die Tachykinine und CGRP einerseits gehören zum exitatorischen NANC-System (eNANC) [47], NOS, VIP und NPY andererseits zum inhibitorischen NANC-System (i-NANC) [48]. In den letzten Jahren erlangten die NANC-Mediatoren immer mehr an Bedeutung, da sie möglicherweise an der Pathogenese des Asthmas bronchiale beteiligt sind ( $\bullet$ Tab. 1).

\section{Tachykinine}

Bei den Tachykininen handelt es sich um eine Familie von Neuropeptiden, die alle an ihrem C-terminalen Ende die gleiche Aminosäuresequenz als aktiven Teil besitzen (Phe-X-Gly-Leu-Met-NH2)
[49]. Die Tachykinine gehören zu der Gruppe der nicht-adrenergen nicht-cholinergen Transmitter (NANC-System) und sind proimflammatorisch. Historisch wurde ihnen der Name wegen ihrer Fähigkeit gegeben, schnell eine Kontraktion von Darmmuskeln hervorzurufen. In den Atemwegen kommen neben Substance $P$ (SP) weitere Tachykinine wie Neurokinin A (NKA) sowie dessen N-terminal verlängerte Peptide Neuropeptid K (NPK) [50] und Neuropeptid $\gamma(\mathrm{NPY})$ [51] vor. Diese Peptide werden von gleichen Genen codiert, durch unterschiedliches Splicen und Schneiden sowie Falten des Vorläuferproteins (Präprotachykinin) entstehen die vier Tachykinine: SP, Neurokinin A, B, K [52]. Weitere Tachykinine wie Virokinin und Hemokinin wurden vor kurzem entdeckt und charakterisiert [53].

Tachykinine werden von den Nervenfasern der unteren Atemwege der Maus, des Meerschweinchens und des Menschen synthetisiert $[23,54]$. Ort der Tachykininsynthese sind sensible Neurone der vagalen Ganglien $[5,55]$ und der thorakalen Spinalganglien [56], deren Axone sich in der gesamten Lunge verteilen. Ihre Wirkungen vermitteln die Tachykinine über Neurokinin-1 (NK-1)-, Neurokinin-2(NK-2)- und Neurokinin-3(NK 3)-Rezeptoren [57]. Es findet sich ein Muster der Verteilung der einzelnen Rezeptoren, so ist NK-1 im Bereich von Gefäßen, Drüsen und dem Epithel lokalisiert. SP bindet vornehmlich an den NK-1-Rezeptor. Der NK-2-Rezeptor liegt auf den glatten Muskeln der Atemwege und NKA besitzt die höchste Affinität zu diesem Rezeptor $[58,59]$. Die Wirkungen von Tachykininen in den Atemwegen sind proinflammatorisch und tragen zu einer Verstärkung der allergischen Atemwegsentzündung wie bei Asthma bronchiale bei.

\section{Calcitonin Gene-verwandtes Peptid (CGRP)}

CGRP gehört zu den exzitatorischen Neurotransmittern des NANC-Systems. Es wird im Lungengewebe in Umgebung von Gefäßen, glatter Muskulatur und dem Epithel gefunden. CGRP ist häufig kolokalisiert mit Tachykininen $[60,61]$. Weiterhin ist CGRP in neuroendokrinen Zellklustern nachweisbar, welche durch parasympatische Nerven kontrolliert werden und im Bereich der Alveolen bis Bronchien nachweisbar sind. Diese spielen bei Stress-induzierter Lungenschädigung eine wichtige Rolle [62].

Der CGRP-Rezeptor gehört zur G-Protein-Familie [63] und die Wirkung des CGRP ist als konzentrationsabhängig beschrieben [64]. So können in vitro Bronchokonstriktion und -dilatation ver-

\begin{tabular}{|c|c|c|c|c|}
\hline $\begin{array}{l}\text { Funktionelles System } \\
\text { Trachea }\end{array}$ & Herkunft & Klass. Transmitter & NANC-Mediatoren & \multirow{8}{*}{$\begin{array}{l}\text { Tab. } 1 \text { Vorkommen von } \\
\text { Mediatoren in sympathischen, } \\
\text { parasympathischen und sensi- } \\
\text { blen Ganglien der unteren } \\
\text { Atemwege und der Lunge. }\end{array}$} \\
\hline sympathisch & $\begin{array}{l}\text { Ggl. cervicale superius } \\
\text { Ggl s stellatum }\end{array}$ & NA & $\begin{array}{l}\text { NPY, VIP, NO } \\
\text { Dynorphin }\end{array}$ & \\
\hline parasympathisch & lokale Ggll. & ACh & VIP, NO, Enkephalin & \\
\hline sensibel & $\begin{array}{l}\text { Ggl. nodosum } \\
\text { Ggl. jugulare } \\
\text { Spinalggl.? }\end{array}$ & $\begin{array}{l}\text { Aspartat } \\
\text { Glutamat }\end{array}$ & $\begin{array}{l}\text { SP, NKA, CGRP } \\
\text { NO, Dynorphin }\end{array}$ & \\
\hline \multicolumn{4}{|l|}{ Lunge } & \\
\hline sympathisch & $\begin{array}{l}\text { Ggl. cervicale superius } \\
\text { Ggl. stellatum } \\
\text { Grenzstranggl. T3-T5 }\end{array}$ & NA & $\begin{array}{l}\text { NPY, VIP, NO } \\
\text { Dynorphin }\end{array}$ & \\
\hline parasympathisch & lokale Ggll. & $\mathrm{ACh}$ & VIP, NO, Enkephalin & \\
\hline sensibel & $\begin{array}{l}\text { Ggl. nodosum } \\
\text { Ggl. jugulare } \\
\text { Spinalggl. C7-T }\end{array}$ & $\begin{array}{l}\text { Aspartat } \\
\text { Glutamat }\end{array}$ & $\begin{array}{l}\text { SP, NKA, CGRP, } \\
\text { NO, Dynorphin }\end{array}$ & \\
\hline
\end{tabular}


ändert werden. Bei Epithelschaden, wie es bei Atemwegserkrankungen vorkommen kann, überwiegt immer die Konstriktion. Weiterhin konnte gezeigt werden, dass durch CGRP in hoher Konzentration eine Depolarisation von parasympathischen Nerven ausgelöst wird und bei geringen Konzentrationen eine Hyperpolarisation verursacht wird [65]. Somit wird der Einfluss von CGRP bei chronisch entzündlichen Erkrankungen klar. Eine Akkumulation von CGRP wurde in Tiermodellen des Asthma bronchiale nachgewiesen, eine 14-fach erhöhte Konzentration lag in der Lunge und in den Ganglien vor. Wie auch beim Asthma muss eine Langzeit-Akkumulation beim COPD angenommen werden [66].

\section{Inhibitorische NANC-Mediatoren: VIP/PACAP}

Vasoaktives Intestinales Polypeptid (VIP) ist ein Neuropeptid der sympathischen und parasympathischen Nervenfasern. Histochemisch ist es im Bereich von tracheobronchialen Muskeln, Drüsen und kleinen Gefäßen lokalisiert [67]. In einem Tiermodell konnte gezeigt werden, dass in geringer Konzentration VIP die Freisetzung von ACh hemmt [68]. Bei Patienten mit Asthma und COPD wurde eine geringere Plasmakonzentration von VIP als antiinflammatorischer Mediator gemessen, wohingegen CGRP-, SPund NPY-Spiegel deutlich erhöht waren. Eine Korrelation zwischen dem Plasmaspiegel besteht zwischen VIP und der Reversibilität der Atemwegsobstruktion [69]. VIP ist vor allem ein Broncho- und Vasodilatator [70].

Pituitary Adenylate Cyclase-activating Polypeptid (PACAP) ist ebenfalls ein Neurotransmitter mit bronchodilatatorischen Effekten [71]. Es bewirkt eine Hemmung der IL-2-Freisetzung und könnte in Zukunft einen neuen Ansatz der medikamentösen Beeinflussung von Atemwegsobstruktionen bieten [72]. Die bei der COPD vorliegende pathologische Verschiebung vom Proteasen/ Antiproteasen-Gleichgewicht wird durch einen protektiven Effekt von VIP/PACAP nach Untersuchungen von Onoue et al. vermindert. Die Induktion von Caspasen und Metalloproteasen wird vermindert [73] .

\section{Neuropeptid Tyrosin (NPY)}

NPY ist ein aus 36 Aminosäuren aufgebautes Peptid [74]. Es gehört zusammen mit Peptid YY zu der großen Familie der pankreatischen Polypeptide. NPY kommt in hohen Konzentrationen im Gehirn vor, während Peptid YY vorwiegend im gastrointestinalen Trakt nachgewiesen wird. In den Atemwegen der Katze, des Meerschweinchens, der Ratte [15], der Maus [34,75] und des Menschen konnten zahlreiche Nervenfasern mit NPY-Immunreaktivität nachgewiesen werden. Nervenfasern mit NPY finden sich reichlich um die Blutgefäße, seromukösen Drüsen, in der Tunica mucosa und der glatten Muskulatur der tracheobronchialen Wand. Eine gehäufte Kolokalisation von NPY mit TH in Nervenfasern, die die Lunge innervieren, wurde mit immunohistochemischen Färbungen gefunden. Tracing-Studien am Meerschweinchen und an der Maus zeigten, dass diese NPY- und TH-positiven Nervenfasern aus den sympathischen Ganglien wie dem Ganglion cervicale superius und Ganglion stellatum stammen [5]. Die Wirkungen von NPY und Peptid YY werden über NPY-Rezeptoren vermittelt. Die zentrale Wirkung von NPY liegt wahrscheinlich in der Regulation des Blutdrucks und der Nahrungsaufnahme $[5,76]$. NPY relaxiert die glatte Atemwegsmuskulatur, führt aber zu einer Vasokonstriktion der Blutgefäße der Atemwege und ist an der Regulation der Drüsensekretion beteiligt [77] ( Tab. 1).

\section{Veränderung der Atemwegsinnervation unter pathophysiologischen Bedingungen $\nabla$}

\section{Autonome Innervation der unteren Atemwege}

Die Stimulation der sympathischen Atemwegsinnervation führt, vermittelt über die Effekte auf die glatte bronchiale Muskulatur, die bronchialen Gefäße und Drüsen, zu einer Erweiterung der Bronchien und Bronchiolen. Im Gegensatz zu anderen Spezies sind Effekte der sympathisch-adrenergen Atemwegsinnervation auf den Tonus der glatten Muskulatur der Atemwege des Menschen gering ausgeprägt [78]. Eine Reihe von $\beta 2$-Rezeptorenagonisten, wie Sabutamol, Fenoterol, Reprotanol, Terbutalin, gehören zu den effektivsten Bronchodilatatoren und sind fester Bestandteil der Medikation in der Behandlung von bronchialer Obstruktion wie bei Asthma bronchiale. Bei der Maus konnte zum ersten Mal gezeigt werden, dass viele sympathische Neurone der Atemwege aus dem SCG und Stellatum NA oder/und NPY produzieren. Nach der Sensibilisierung und Provokation mit einem Allergen wurde allerdings keine signifikante Veränderung der Anzahl dieser Neuronen gefunden [34]. NPY ist in der Lage, die Hauptsymptome wie Niesen, Juckreiz und Hypersekretion bei Rhinitis zu lindern [79]. Weiterhin moduliert NPY die cholinerge Neurotransmission und verhindert Tachykininfreisetzung aus sensiblen Nerven. Bei Patienten mit Asthma bronchiale wurden nach Exazerbation erhöhte NPY-Plasmaspiegel gefunden. Bioptisch kommen allerdings weniger NPY-positive Nervenfasern bei Patienten mit Asthma bronchiale und COPD im Vergleich zu den Gesunden vor [80,81]. Daher wurde eine protektive Rolle von NPY bei chronisch entzündlichen und obstruktiven Atemwegserkrankungen vermutet. Wie oben bereits beschrieben, kommt auch VIP in sympathischen Nerven vor. Diesem kommt wie auch dem PACAP eine antiinflammatorische und broncho- und vasodilatatorische Rolle zu. Die Nutzbarkeit dieser Eigenschaften ist nach der aktuellen Datenlage eher zurückhaltend anzusehen. Die kurze Halbwertszeit von VIP und die Nichtüberlegenheit gegenüber Sultanol verschlechtern die Chancen einer weiteren Entwicklung [67].

Bei Asthma bronchiale und COPD spielt der Parasympathikus in der Lunge eine bedeutende Rolle. Hier kommt es durch Aktivierung des Parasympathikus zu einer Bronchokontraktion und Einengung der Atemwege.

Unter normalen Bedingungen arbeitet der Sympathikus und Parasympathikus koordiniert in einem Gleichgewicht. Bei einem Asthmaanfall kommt es zu einer Dominanz der parasympathischen Innervation. Das Ungleichgewicht zwischen der sympathischen und parasympathischen Innervation führte zu einer Bronchokontraktion mit der Einengung der Atemwege. Bei einer Exazerbation von Asthma bronchiale oder COPD kann eine erhöhte Aktivität des Parasympathikus zu einer Bronchokontraktion führen. Ein besseres Verständnis der parasympathischen Innervation könnte helfen, effektivere Behandlungen für Asthma und COPD zu entwickeln $[42,43]$ ( $\bullet$ Abb. 3).

\section{Sensible Innervation unter pathologischen Bedingungen} Wie oben bereits formuliert, kommt es durch die Reizung von sensiblen Neuronen zu einer Freisetzung von Neurotransmittern im Bereich der Dendriten und im Bereich des Somas (Ganglion). Um die Rolle von Tachykininen bei der allergischen Atemwegsentzündung zu überprüfen, erfolgte die Bestimmung der Expression von Tachykininen im Asthmamodell der Maus [55, 82 - 85]. Pharmakologische Studien mit NK-1-Rezeptor-Antagonisten lieferten den entsprechenden Nachweis für eine Beteiligung von 
Tachykininen an pathologischen Veränderungen der Atemwege [86]. Die Beteiligung neuronaler Tachykinine an der allergischen Atemwegsentzündung wurde bei vielen Spezies nachgewiesen. Unter normalen Bedingungen werden Tachykinine ausschließlich in kleinen sensiblen Neuronen mit einem Durchmesser von weniger als $20 \mu \mathrm{m}$ gefunden. Bei der allergischen Atemwegsentzündung wurden Tachykinine in atemwegsspezifischen Neuronen mit größerem Durchmesser gefunden. Die Induktion von Tachykininen in sensiblen Neuronen wurde bereits beim Meerschweinchen nach Allergensensibilisierung und Provokation beobachtet [82]. Ähnlich wie beim Meerschweinchen wurde die Induktion von Tachykininen in sensiblen atemwegsspezifischen Neuronen mit myelinisierten Axonen (Aס-Fasern) (die hohe Leitungsgeschwindigkeit erreichen können) bei der Maus nachgewiesen [85]. Aufgrund der morphologischen Kolokalisation von SP mit TRPV1 oder trkA in atemwegsspezifischen Spinalganglienneuronen wird eine Beteiligung der sensiblen spinalen Atemwegsinnervation an der neurogenen Entzündung von Atemwegserkrankungen wie Asthma bronchiale und COPD vermutet [83,87-90]. CGRP führt bei Reizungen der sensiblen Nervenfasern zu einer Atemwegsentzündung.

Nicht nur lokal im Bereich der sensiblen Nervenendigungen kommt es bei Reizungen zu einer Modulation der Entzündung. Im Bereich des sensiblen Zellkörpers kann bei Depolarisation eine Freisetzung von Tachykininen erfolgen. Eine Einflussnahme ist auf sowohl para- wie auch sympathische Neuronen möglich. Dies wird als der periphere sensible Reflex beschrieben. Weiter kommt es über Interneuronen zur Mitregulation der parasympatischen oder sympathischen Reaktion und Innervation, wobei dieser Weg als einziger nicht auf der Freisetzung von parakriner NANC-Freisetzung beruht [91].

Die durch proinflammatorische Neuropeptide induzierte Entzündung wurde als neurogene Entzündung erstmals in den sechziger Jahren beschrieben. Es wurde nachgewiesen, dass eine Denervierung oder eine Vorbehandlung mit Capsaicin die neurogene Entzündung abschwächen konnte [92].

Pharmakologische Studien mit NK- und TRPV1-Antagonisten wie Capsazepine konnten zeigen, dass Neuropeptide wie SP und CGRP aus den Nervenfasern an der Pathogenese entzündlicher Prozesse wie bei den Ethanol-induzierten Entzündungen in den Atemwegen beteiligt sind [93]. Es konnte nachgewiesen werden, dass die durch Ethanol induzierte Bronchokonstriktion und Plasmaextravasation in den Atemwegen des Meerschweinchens durch die Vorbehandlung mit Capsaicin oder mit Tachykinin-Rezeptorantagonisten oder mit spezifischen TRPV1-Antagonisten wie Capsazepine verhindert werden können [94] ( $\bullet$ Abb. 3).

Die neurogene Entzündung kann als eine komplexe Reaktion, die bei vielen Lungenerkrankungen vorkommt, bestehend aus gesteigerter Gefäßpermeabilität, Plasmaextravasation, Schleimsekretion, Einwanderung von Entzündungszellen, und Gefäßremodelling bewirkt, durch die lokale Freisetzung proinflammatorischer Mediatoren, beschrieben werden [91].

Tachykinine (eingeschlossen Virokinine und Hemokinine) werden nach ihrer Freisetzung von Neutraler Endopeptidase (NEP) und einem Angiotensin-umwandelnden Enzym abgebaut. Mit der Entwicklung von neuen hochwirksamen spezifischen tachykinergen und non-tachykinergen Rezeptorantagonisten erhofft man sich, die entzündlichen Erkrankungen wie Asthma bronchiale und allergische Rhinitis in Zukunft spezifischer therapieren zu können [95 -97]. Bis heute erfolgte nur eine erfolgreiche Anwendung von NK-1/NK-2- oder NK-1/NK-2/NK-3-Antagonisten bei Tieren und nicht beim Menschen. Im Tiermodell konnte durch die Behandlung von NK-1/NK-2- oder NK-1/NK-2/NK-3Antagonisten eine Bronchokonstriktion verhindert werden. Noch kann keine Aussage über die Effektivität der TachykininAgonisten beim Menschen getroffen werden [59].

\section{Interaktion zwischen Zellen des Nerven- und des Immunsystems bei chronisch entzündlichen und obstruktiven Atemwegserkrankungen $\nabla$}

Unter physiologischen Bedingungen wird Neurotrophin primär von Neuronen und nervenverwandten Zellen wie Gliazellen und Schwannzellen $[98,99]$ und auch von einer Reihe von immunkompetenten Zellen, einschließlich Mastzellen [100], Eosinophilen [101], T-Zellen und B-Zellen [102], synthetisiert. Immunzellen können wiederum als Zielzellen von Neurotrophinen dienen. Der Nervenwachstumsfaktor (NGF) wurde erhöht in allergischen Entzündungen gefunden $[103,104]$. Immunohistochemische Färbungen zeigten, dass sensible Atemwegsneuronen Neurotrophinrezeptoren wie trkA, trkB und p75 Neurotrophin-Rezeptor in ihren Zellkörpern und Nervenfasern exprimieren [105]. Aktivierung dieser Rezeptoren können Veränderungen an den Neuronen hervorrufen. NGF war in der Lage, 24 Stunden nach der Injektion in den Hauptbronchus nicht nur beim Meerschweinchen, sondern auch bei der Maus, Tachykininsynthese in den Atemwegsneuronen zu induzieren [106]. Es verdichten sich die Hinweise, dass die Neurotrophine als Signalmoleküle bei der neuroimmunologischen Interaktion eine Schlüsselrolle einnehmen und dass sie darüber hinaus einen immunologischen Einfluss auf die sensiblen Atemwegsneuronen ausüben.

\section{Danksagung \\ $\nabla$}

Diese Arbeit wird durch die DFG im Rahmen des Projektes DFGDI 1429 und des Graduiertenkollegs 1441: Regulation der allergischen Entzündung in Lunge und Haut an PD Dr. Q. Thai Dinh und Prof. Dr. Tobias Welte gefördert.

\section{Interessenkonflikt \\ $\nabla$}

Die Autoren geben an, dass kein Interessenkonflikt besteht.

\section{Institute}

Zentrum für Innere Medizin, Medizinische Hochschule Hannover (MHH), Klinik für Pneumologie, Hannover (Leiter: Prof. Dr. T. Welte)

${ }^{2}$ Charité Zentrum 12 für Innere Medizin und Dermatologie, Medizinische Klinik mit Schwerpunkt Psychosomatik, Charité - Universitätsmedizin Berlin, Freie Universität Berlin \& Humboldt-Universität zu Berlin, Berlin (Leiter: Prof. Dr. B. F. Klapp)

3 Zentrum für Pädiatrie, Klinische Forschergruppe Allergologie, Charité Universitätsmedizin Berlin, Freie Universität Berlin \& Humboldt-Universität zu Berlin, Berlin (Leiter: Prof. Dr. A. Fischer)

4 Fraunhofer Institut für Toxikologie und Experimentelle Medizin (ITEM), Abteilung Immunologie, Allergologie und Immuntoxikologie, Hannover (Leiter: Prof. Dr. N. Krug) 


\section{Literatur}

1 Barnes PJ. Asthma as an axon reflex. Lancet 1986; 1: 242-245

2 Barnes PJ. Mediators of chronic obstructive pulmonary disease. Pharmacol Rev 2004; 56: 515-548

3 Willis T. Cap. XI: Opera omnia. De asthmate. Band II, Sect. 11681

4 Lundberg JM, Hemsen A, Larsson $O$ et al. Neuropeptide Y receptor in pig spleen: binding characteristics, reduction of cyclic AMP formation and calcium antagonist inhibition of vasoconstriction. Regul Pept 1988; 20: $125-139$

5 Kummer W, Fischer A, Kurkowski R et al. The sensory and sympathetic innervation of guinea-pig lung and trachea as studied by retrograde neuronal tracing and double-labelling immunohistochemistry. Neuroscience 1992; 49: 715-737

6 Pisi G, Olivieri D, Chetta A. The airway neurogenic inflammation: Clinical and pharmacological implications. Inflamm Allergy Drug Targets 2009; 8: 176 - 181

7 Mann SP. The innervation of mammalian bronchial smooth muscle: The localization of catecholamines and cholinesterase. Histochemistry $1971 ; 3: 319-331$

8 Doidge JM, Satchell DG. Adrenergic and nonadrenergic inhibitory nerves in mammalian airways. J Auton Nervous System 1982; 5: 83-99

9 Van der Velden VH, Hulsmann AR. Autonomic innervation of human airways: structure, function, and pathophysiology in asthma. Neuroimmunomodulation 1999; 6: 145 - 159

10 Sheppard MN, Kurian SS, Henzen-Logmans SC et al. Neurone-specific enolase and S-100: new markers for delineating the innervation of the respiratory tract in man and other mammals. Thorax 1983; 38: $333-340$

11 Barnes PJ, Chung KF, Page CP et al. Inflammatory mediators of asthma: an update. Pharmacol Rev 1998; 50: 515-596

$12 \mathrm{Kim}$ YD, Lee SH, Lee SY et al. The effect of thoracosopic thoracic sympathetomy on pulmonary function and bronchial hyperresponsiveness. J Asthma 2009; 46: 276-279

13 Barnes P, Karliner J, Hamilton C et al. Demonstration of alpha 1-adrenoceptors in guinea pig lung using 3H-prazosin. Life Sci 1979; 25: $1207-1214$

14 Lundberg JM, Terenius L, Hokfelt T et al. High levels of neuropeptide Y in peripheral noradrenergic neurons in various mammals including man. Neurosci Lett 1983; 42: 167-172

15 Uddman R, Sundler F, Emson P. Occurrence and distribution of neuropeptide-Y-immunoreactive nerves in the respiratory tract and middle ear. Cell Tissue Res 1984; 237: 321 - 327

16 Bowden JJ, Gibbins IL. Vasoactive intestinal peptide and neuropeptide $\mathrm{Y}$ coexist in non-noradrenergic sympathetic neurons to guinea pig trachea. J Auton Nerv Syst 1992; 38: 1-19

17 Fischer A, Mundel P, Mayer B et al. Nitric oxide synthase in guinea pig lower airway innervation. Neurosci Lett 1993; 149: 157-160

18 Myers A, Weinreich D, Undem BJ et al. A reidentifiable parasympathetic ganglion on the bronchus of the guinea pig: anatomical and electrophysiological characteristics. Fedii Am Socs exp Biol J 1988; 2: A1056

19 Myers AC, Undem BJ, Weinreich D. Electrophysiological properties of neurons in guinea pig bronchial parasympathetic ganglia. Am J Physiol 1990; 259: L403 - L409

20 Kalia M, Mesulam MM. Brainstem projections of sensory and motor components of the vagus complex in the cat: II. Laryngeal tracheobronchial, pulmonary, cardial and gastrointestinal branches. J comp Neurol 1980; 193: 467-508

21 Kalia M, Mesulam MM. Brain stem projections of sensory and motor components of the vagus complex in the cat: I. The cervical vagus and nodose ganglion. J Comp Neurol 1980; 193: 435-465

22 Partanen M, Laitinen A, Hervonen A et al. Catecholamine- and acetylcholinesterase-containing nerves in human lower respiratory tract. Histochemistry 1982; 76: 175-188

23 Lundberg JM, Hokfelt T, Martling CR et al. Substance P-immunoreactive sensory nerves in the lower respiratory tract of various mammals including man. Cell Tissue Res 1984; 2352: 251 - 261

24 Canning BJ, Fischer A. Localization of cholinergic nerves in lower airways of guinea pigs using antisera to choline acetyltransferase. Am J Physiol 1997; 272: L731 - L738

25 Dey RD, Shannon WA, Said ST et al. Localization of VIP-immunoreactive nerves in airways and pulmonary vessels of dogs, cat, and human subjects. Cell Tissue Res 1981; 220: 231 - 238
26 Fischer A, Canning BJ, Kummer W. Correlation of vasoactive intestinal peptide and nitric oxide synthase with choline acetyltransferase in the airway innervation. Ann N Y Acad Sci 1996a; 805: 717-722

27 Dey R, Hoffpauir J, Said SI. Co-localisation of vasoacitve intestinal peptide- and substance P-containing nerves in cat bronchi. Neuroscience 1988; 24: $275-281$

28 Fontan JJ, Cortright DN, Krause JE et al. Substance P and neurokinin-1 receptor expression by intrinsic airway neurons in the rat. Am J Physiol Lung Cell Mol Physiol 2000; 278: L344 - L355

29 Nohr D, Eiden LE, Weihe E. Coexpression of vasoactive intestinal peptide, calcitonin gene-related peptide and substance $\mathrm{P}$ immunoreactivity in parasympathetic neurons of the rhesus monkey lung. Neurosci Lett 1995; 199: $25-28$

30 Shimosegawa T, Foda HD, Said SI. [Met]enkephalin-Arg6-Gly7-Leu8immunoreactive nerves in guinea-pig and rat lungs: distribution, origin, and co-existence with vasoactive intestinal polypeptide immunoreactivity. Neuroscience 1990; 36: 737-750

31 Ricco MM, Kummer W, Biglari B et al. Interganglionic segregation of distinct vagal afferent fibre phenotypes in guinea-pig airways. J Physiol (Lond) 1996; 496: 521 - 530

32 Barnes PJ. Neurogenic inflammation in the airways. Respir Physiol $2001 ; 125-2: 145-154$

33 Kuo YL, Lai CJ. Ovalbumin sensitizes vagal pulmonary C-fiber afferents in Brown Norway rats. J Appl Physiol 2008; 105: 611-620

34 Taylor-Clark TE, McAlexander MA, Nassenstein C. Relative contributions of TRPA1 and TRPV1 channels in the activation of vagal bronchopulmonary C-fibres by the endogenous autacoid 4-oxononenal. J Physiol 2008; 15; 586: 3447-3459

35 Solway J, Leff AR. Sensory neuropeptides and airway function. J Appl Physiol 1991; 71: 2077-2087

36 Verastegui C, Prada Oliveira A, Fernandez-Vivero J et al. Calcitonin gene-related peptide immunoreactivity in adult mouse lung. Eur J Histochem 1997; 41: 119-126

37 Dinh QT, Groneberg DA, Witt C et al. Expression of Tyrosine Hydroxylase and Neuropeptide Tyrosine in Mouse Sympathetic Airwayspecific Neurons under Normal Situation and Allergic Airway Inflammation. Clin Exp Allergy 2004; 34: 1934-1941

38 Kummer W, Bachmann S, Neuhuber WL et al. Tyrosine-hydroxylasecontaining vagal afferent neurons in the rat nodose ganglion are independent from neuropeptide-Y-containing populations and project to esophagus and stomach. Cell Tissue Res 1993; 271: 135-144

39 Coburn RF, Tomita T. Evidence for nonadrenergic inhibitory nerves in the guinea pig trachealis muscle. Am J Physiol 1973; 224: 1072 - 1080

40 Gosens R, Zaagsma J, Meurs H, Halayko AJ. Muscarinic receptor signaling in the pathophysiology of asthma and COPD. Respir Res 2006; 9; 7: 73

41 Gross NJ. Anticholinergic agents in asthma and COPD. Eur J Pharmacol 2006; 533: 36 - 39

42 Belmonte KE. Cholinergic pathways in the lungs and anticholinergic therapy for chronic obstructive pulmonary disease. Proc Am Thorac Soc 2005; 2: 297-304

43 Gross NJ, Skorodin MS. Role of the parasympathetic system in airway obstruction due to emphysema. N Engl J Med 1984; 16; 311: 421 425

44 Kummer W, Lips KS, Pfeil $U$. The epithelial cholinergic system of the airways. Histochem Cell Biol 2008; 130: 219-234

45 Boichot E, Lagente V, Paubert-Braquet $M$ et al. Inhaled substance P induces activation of alveolar macrophages and increases airway responses in the guinea-pig. Neuropeptides 1993; 25: 307-313

46 Widdicombe JG. Autonomic regulation. i-NANC/e-NANC. Am J Respir Crit Care Med 1998; 158: 171 - 175

47 Karlsson JA, Finney MJ, Persson CG et al. Substance P antagonists and the role of tachykinins in non-cholinergic bronchoconstriction. Life Sci 1984; 35: $2681-2691$

48 Li CG, Rand MJ. Evidence that part of the NANC relaxant response of guinea-pig trachea to electrical field stimulation is mediated by nitric oxide. Br J Pharmacol 1991; 102: 91 - 94

49 Chang M, Leeman SE, Niall HD. Amino-acid sequence of substance P. Nat New Biol 1971; 232: 86-87

50 Tatemoto K, Lundberg JM, Jornvall H, Mutt V. Neuropeptide K: isolation, structure and biological activities of a novel brain tachykinin. Biochem Biophys Res Commun 1985; 128: 947-953

51 Kage R, McGregor GP, Thim L et al. Neuropeptide-gamma: a peptide isolated from rabbit intestine that is derived from gamma-preprotachykinin. J Neurochem 1988; 50: 1412-1417 
52 Kotani $Y$, Hirota $Y$, Suguyama $K$ et al. Effects of noxious stimuli and anesthetic agents on substance $P$ content in rat central nervous system. Jpan J Pharmacol 1986; 40: $143-147$

53 Groneberg DA, Harrison S, Dinh QT et al. Tachykinins in the respiratory tract. Curr Drug Targets 2006; 7: 1005 - 1010

54 Hua XY, Theodorsson-Norheim E, Brodin E et al. Multiple tachykinins (neurokinin A, neuropeptide $\mathrm{K}$ and substance $\mathrm{P}$ ) in capsaicin-sensitive sensory neurons in the guinea-pig. Regul Pept 1985; 3: 1-19

55 Dinh QT, Groneberg DA, Peiser C et al. Expression of substance P and nitric oxide synthase in vagal sensory neurons innervating the mouse airways. Regul Pept 2005; 126: 189 - 194

56 Dinh QT, Groneberg DA, Peiser C et al. Nerve growth factor-induced substance $P$ in capsaicin-insensitive vagal neurons innervating the lower mouse airway. Clin Exp Allergy 2004; 34: 1474-1479

57 Helke CJ, Krause JE, Mantyh PW et al. Diversity in mammalian tachykinin peptidergic neurons: multiple peptides, receptors, and regulatory mechanisms. Faseb J 1990; 4: 1606 - 1615

58 Komatsu T, Yamamoto M, Shimokata K et al. Distribution of substance P-immunoreactive and calcitonin gene-related peptide-immunoreactive nerves in normal human lungs. Int Arch Allergy Appl Immunol 1991; 95: $23-28$

59 Joos GF, Germonpré PR, Pauwels RA. Role of tachykinins in asthma. Allergy 2000; 55: 321 - 337

60 Hua XY, Theodorsson-Norheim E, Lundberg JM et al. Co-localization of tachykinins and calcitonin gene-related peptide in capsaicin-sensitive afferents in relation to motility effects on the human ureter in vitro. Neuroscience 1987; 23: $693-703$

61 Van Rossum D, Hanisch UK, Quirion R. Neuroanatomical localization, pharmacological characterization and functions of CGRP, related peptides and their receptors. Neurosci Biobehav Rev 1997; 21: 649-678

62 Adriaensen D, Scheuermann DW, Gajda M et al. Functional implications of extensive new data on the innervation of pulmonary neuroepithelial bodies. Ital J Anat Embryol 2001; 106: 395-403

63 Van Rossum D, Hanisch UK, Quirion R. Neuroanatomical localization, pharmacological characterization and functions of CGRP, related peptides and their receptors. Neurosci Biobehav Rev 1997; 21: 649-678

64 Springer J, Geppetti P, Fischer A. Calcitonin gene-related peptide as inflammatory mediator. Pulm Pharmacol Ther 2003; 16: 121-130

65 Kajekar R, Myers AC. Calcitonin gene-related peptide affects synaptic and membrane properties of bronchial parasympathetic neurons. Respir Physiol Neurobiol 2008; 1; 160: 28-36

66 Tsukiji J, Sango K, Udaka N. Long-term induction of beta-CGRP mRNA in rat lungs by allergic inflammation. Life Sci 2004; 26; 76: 163-177

67 Groneberg DA, Rabe KF, Fischer A. Novel concepts of neuropeptide-based drug therapy: vasoactive intestinal polypeptide and its receptors. Eur J Pharmacol 2006; 8; 533: $182-194$

68 Aizawa $H$, Inoue $H$, Shigyo $M$ et al. VIP antagonists enhance excitatory cholinergic neurotransmission in the human airway. Lung 1994; 172: $159-167$

69 Cardell LO, Uddman R, Edvinsson L. Low plasma concentrations of VIP and elevated levels of other neuropeptides during exacerbations of asthma. Eur Respir J 1994; 7: 2169-2173

70 Schmidt DT, Rühlmann E, Waldeck B et al. The effect of the vasoactive intestinal polypeptide agonist Ro $25-1553$ on induced tone in isolated human airways and pulmonary artery. Naunyn Schmiedebergs Arch Pharmacol 2001; 364: 314-320

71 Kinhult J, Uddman R, Cardell LO. The induction of carbon monoxidemediated airway relaxation by PACAP 38 in isolated guinea pig airways. Lung 2001; 179: $1-8$

72 Yoshihara Y, Tsukazaki T, Osaki M et al. Altered expression of inflammatory cytokines in primary osteoarthritis by human $T$ lymphotropic virus type I retrovirus infection: a cross-sectional study. Arthritis Res Ther 2004; 6: R347-R354

73 Onoue S, Ohmori Y, Endo K et al. Vasoactive intestinal peptide and pituitary adenylate cyclase-activating polypeptide attenuate the cigarette smoke extract-induced apoptotic death of rat alveolar L2 cells. Eur J Biochem 2004; 271: 1757 - 1767

74 Tatemoto K, Carlquist M, Mutt V. Neuropeptide Y- a novel brain peptide with structural similarities to peptide YY and pancreatic polypeptide. Nature 1982; 296: 659-660

75 Verastegui C, Fernandez-Vivero J, Prada A et al. Presence and distribution of 5HAT-,VIP-, NPY-, and SP-immunoreactive structures in adult mouse lung. Histol Histopathol 1997; 12: $909-918$
76 Bing C, King P, Pickavance L et al. The effect of moxonidine on feeding and body fat in obese Zucker rats: role of hypothalamic NPY neurones. Br J Pharmacol 1999; 127: 35-42

77 Lundberg JM, Modlin A. Neuropeptid Y in the airways. Neuropeptides in Respiratory Medicine. New York: Marcel Dekker, 1994: 161 - 172

78 De Jongste JC, Jongejan RC, Kerrebijn KF. Control of airway caliber by autonomic nerves in asthma and in chronic obstructive pulmonary disease. Am Rev Respir Dis 1991; 143: 1421 - 1426

79 Lacroix JS, Mosimann BL. Attenuation of allergen-evoked nasal responses by local pretreatment with exogenous neuropeptide $\mathrm{Y}$ in atopic patients. J Allergy Clin Immunol 1996; 98: 611 - 616

80 Cardell LO, Uddman R, Edvinsson L. Low plasma concentrations of VIP and elevated levels of other neuropeptides during exacerbations of asthma. Eur Respir J 1994; 7: 2169-2173

81 Chanez P, Springall D, Vignola AM et al. Bronchial mucosal immunoreactivity of sensory neuropeptides in severe airway diseases. Am J Respir Crit Care Med 1998; 158: 985 - 990

82 Fischer A, McGregor GP, Saria A et al. Induction of tachykinin gene and peptide expression in guinea pig nodose primary afferent neurons by allergic airway inflammation. J Clin Invest 1996; 98: 2284-2291

83 Undem BJ, Hunter DD, Liu M et al. Allergen-induced sensory neuroplasticity in airways. Int Arch Allergy Immunol 1999; 118: 150-153

84 Myers AC, Kajekar R, Undem BJ. Allergic inflammation-induced neuropeptide production in rapidly adapting afferent nerves in guinea pig airways. Am J Physiol Lung Cell Mol Physiol 2002; 282: L775 - L781

85 Dinh QT, Mingomataj E, Quarcoo D et al. Allergic airway inflammation induces tachykinin peptides expression in vagal sensory neurons innervating mouse airways. Clin Exp Allergy 2005; 35: 820 - 825

86 Quarcoo D, Schulte-Herbruggen O, Lommatzsch $M$ et al. Nerve growth factor induces increased airway inflammation via a neuropeptidedependent mechanism in a transgenic animal model of allergic airway inflammation. Clin Exp Allergy 2004; 34: 1146-1151

87 Kollarik M, Dinh QT, Fischer A et al. Capsaicin-sensitive and -insensitive vagal bronchopulmonary C-fibres in the mouse. J Physiol 2003; 15 : $869-879$

88 Michael GJ, Averill S, Nitkunan A et al. Nerve growth factor treatment increases brain-derived neurotrophic factor selectively in TrkA-expressing dorsal root ganglion cells and in their central terminations within the spinal cord. J Neurosci 1997; 17: 8476-8490

89 Dinh QT, Groneberg DA, Peiser C et al. Substance P expression in TRPV1 and trkA-positive dorsal root ganglion neurons innervating the mouse lung. Respir Physiol Neurobiol 2004; 144: 15-24

90 De Vries A, Engels F, Henricks PA et al. Airway hyper-responsiveness in allergic asthma in guinea-pigs is mediated by nerve growth factor via the induction of substance P: a potential role for trkA. Clin Exp Allergy 2006; 36: $1192-1200$

91 Barnes PJ. Neurogenic inflammation in the airways. Respir Physiol 2001; $125: 145-154$

92 Jancso N, Jancso-Gabor A, Szolcsanyi J. Direct evidence for neurogenic inflammation and its prevention by denervation and by pretreatment with capsaicin. Br J Pharmacol Chemother 1967; 31: 138-151

93 Trevisani M, Smart D, Gunthorpe MJ et al. Ethanol elicits and potentiates nociceptor responses via the vanilloid receptor- 1 . Nat Neurosci 2002; 5: $546-551$

94 Trevisani M, Gazzieri D, Benvenuti F et al. Ethanol Causes Inflammation in the Airways by a Neurogenic and TRPV1-Dependent Mechanism. J Pharmacol Exp Ther 2004; 309: 1167-1173

95 Dinh QT, Klapp BF, Fischer A. Die sensible Atemwegsinnervation und die Tachykinine bei Asthma und chronisch-obstruktiver Lungenerkrankung (COPD). Pneumologie 2006; 60: 80-85

96 Geppetti P, Materazzi S, Nicoletti P. The transient receptor potential vanilloid 1: role in airway inflammation and disease. Eur J Pharmacol 2006; 533: $207-214$

97 Peiser C, Trevisani M, Groneberg DA et al. Dopamine type 2 receptor expression and function in rodent sensory neurons projecting to the airways. Am J Physiol Lung Cell Mol Physiol 2005; 289: L153 - L158

98 Levi-Montalcini $R$. The nerve growth factor 35 years later. Science 1987; 237; 4819: $1154-1162$

99 Levi-Montalcini R, Skaper SD, Dal Toso $R$ et al. Nerve growth factor: from neurotrophin to neurokine. Trends Neurosci 1996; 11: 514 520

100 Leon A, Buriani A, Dal Toso R et al. Mast cells synthesize, store, and release nerve growth factor. Proc Natl Acad Sci USA 1994; 91; 9: 3739 3743 
101 Noga O, Hanf G, Gorges D et al. Regulation of NGF and BDNF by dexamethasone and theophylline in human peripheral eosinophils in allergics and non-allergics. Regul Pept 2005; 132: 74 - 79

102 Braun A, Lommatzsch M, Mannsfeldt A et al. Cellular sources of enhanced brain-derived neurotrophic factor production in a mouse model of allergic inflammation. Am J Respir Cell Mol Bio 1999; 21: 537-546

103 Nassenstein C, Braun A, Erpenbeck VJ et al. The neurotrophins nerve growth factor, brain-derived neurotrophic factor, neurotrophin-3, and neurotrophin- 4 are survival and activation factors for eosinophils in patients with allergic bronchial asthma. J Exp Med 2003; 198: $455-467$
104 Virchow JC, Julius P, Lommatzsch $M$ et al. Neurotrophins are increased in bronchoalveolar lavage fluid after segmental allergen provocation. Am J Respir Crit Care Med 1998; 158: 2002 - 2005

105 Nassenstein C, Dawbarn D, Pollock K et al. Pulmonary distribution, regulation, and functional role of Trk receptors in a murine model of asthma. J Allergy Clin Immunol 2006; 118: 597-605

106 Hunter DD, Myers AC, Undem BJ. Nerve growth factor-induced phenotypic switch in guinea pig airway sensory neurons. Am J Respir Crit Care 2000; 161: $1985-1990$ 